\title{
STATUS REPORT ON THE DUKE FEL FACILITY*
}

\author{
P.Wang, V. Litvinenko, M. Emamian, J. Faircloth, J. Gustavsson, S. Hartman, S. Mikhailov, P. \\ Morcombe, O. Oakeley, J. Patterson, M. Pentico, I. Pinayev, O. Shevchenko, G. Swift, G. Edwards \\ FEL Laboratory, Duke University, Durham, NC 27708, USA
}

\begin{abstract}
At the Duke Free Electron Laser (FEL) Laboratory, there are two FEL machines: the Mark III infrared FEL and the OK-4/Storage Ring, which produces UV and XUV laser beam as well as gamma rays via Compton backscattering. The recent status of Mark-III machine is described in another paper [1]. Here we will concentrate on the new development of the OK-4/Storage Ring FEL and its performance and capabilities. A brief history of this machine and the future plan are also given in this paper.
\end{abstract}

\section{INTRODUCTION}

The OK-4/Storage Ring FEL is comprised of a 270 $\mathrm{MeV}$ linear accelerator injector, a $1.1 \mathrm{GeV}$ storage ring with OK-4 optical klystron (OK) and two optical beam lines. The ring is housed inside a 25,000 SF hall with the concrete shielding blocks around it. Adjacent to it, there is a newly constructed KECK building with $13,000 \mathrm{SF}$ area dedicated to host users. The $270 \mathrm{MeV}$ injector is located inside a 100 meters long tunnel at the northeast corner of the main building. At present, we have two beam lines connected to the user building and each beam line has a number of beam ports to different user stations. One of the two beam lines is a straight extension of the ring optical cavity, which leads the laser beam and gamma rays penetrating the front mirror to a 60 meters away gamma detector vault.

The $1.1 \mathrm{GeV}$ storage ring is a "third generation" electron ring. It operates with the 18-meter long OK-4 wiggler to serve as a free electron laser facility. It provides a broad variable coherent and spontaneous radiation in the UV and XUV wavelength range. A unique feature is its ability to generate nearly monochromatic and linearly polarized gamma rays by Compton back-scattering inside the optical cavity. It has been serving a worldwide community of nuclear physicists as a variable energy gamma ray source since 1998. By using five pairs of mirrors, this FEL facility can provide a coherent FEL with a tunable wavelength from 193 to 730 nana-meters and gamma ray energy from 2 to $58 \mathrm{MeV}$.

The ring commissioning started on November 1994. The Ok-4 wiggler was installed one year later. The OK-4 lasing and gamma ray generation commenced in November 1996. The user application programs started April 1998. Since then, this facility has served users for approximately half of the time each year and another half of the time for machine up-grade.

\footnotetext{
* Work supported by the DOD MFEL program as administrated by the AFOSR.
}

\section{DESCRIPTION OF THE MACHINE}

The linac injector contains a photo cathode microwave gun, 3 S-band klystrons and 11 SLAC type accelerator sections. With recently up-graded PLC (programmable logic control) system, the injector works rather reliably. The injection energy is $270 \mathrm{MeV}$.

The ring was specially designed to drive the short wavelength FEL. There are three designed features to reach the FEL requirements. They are low emittance, large dynamic aperture and long dispersion free straight sections. The ring emittance and the dynamic aperture are listed below. The two 34-meter long straight sections make the ring a racetrack configuration. One straight section is furnished with OK-4 wiggler and bunching magnet; the other one is occupied by the RF cavity and injection system. The detailed technical design issues have been published in references [2] and [3]. The Table 1 is a summary of the main parameters.

Table 1: operational machine parameters

$$
\begin{array}{ll}
\text { Electron Beam } & \\
\text { Operational energy } & 0.25-1.1 \mathrm{GeV} \\
\text { Ring circumference } & 107.46 \mathrm{~m} \\
\text { RF frequency } & 178.5 \mathrm{Mhz} \\
\text { Dynamic aperture, Ax/Ay } & 56.0 / 16.0 \mathrm{~mm} \mathrm{mrad} \\
\text { Emittance at } 1 \mathrm{GeV}, \varepsilon_{x} / \mathcal{E}_{\mathrm{y}} & 18 / 1.8 \mathrm{~nm} \mathrm{rad} \\
\text { Impedance of ring, } \mathrm{Z} / \mathrm{n} & 2.75+/-0.25 \mathrm{ohm} \\
\text { Beam size in OK-4,Sx/Sy } & 0.27 / 0.085 \mathrm{~mm} \\
\text { OK-4 FEL } & \\
\text { Optical cavity length } & 53.73 \mathrm{~m} \\
\text { Mirror radius } & 27.27 \mathrm{~m} \\
\text { Tuning range } & 193.7-730 \mathrm{~nm} \\
\text { Out-coupled laser power } & 5-300 \mathrm{~mW} \\
\text { Natural line-width } & (1-4) \times 10^{-4} \\
\text { Gain per pass } & \sim 10 \% \\
\text { Compton gamma ray } & \\
\text { Energy range } & 2-58 \mathrm{MeV} \\
\text { Total flux } & 10^{6}-10^{7} / \mathrm{sec} \\
\text { Energy resolution, } & <1 \% \mathrm{FWHM}
\end{array}
$$

The OK-4 contains two electromagnetic wiggler and one bunching magnet. The wiggler period is $10 \mathrm{~cm}$ and the total period number is 67 . The wiggler magnet has a gap of $2.25 \mathrm{~cm}$. There is a field free collision point designed for Compton back-scattering in the center of the optical klystron system.

The two optical mirrors are 53.73 meters apart. The mirror radius is $26.46 \mathrm{~m}$ with a Rayleigh range of $3 \mathrm{~m}$. A 
very sophisticated control and feed-back system have been developed to perform the precise optical alignment and controls [4]. Fig.1 shows the west part of the ring with one wiggler on the left side.

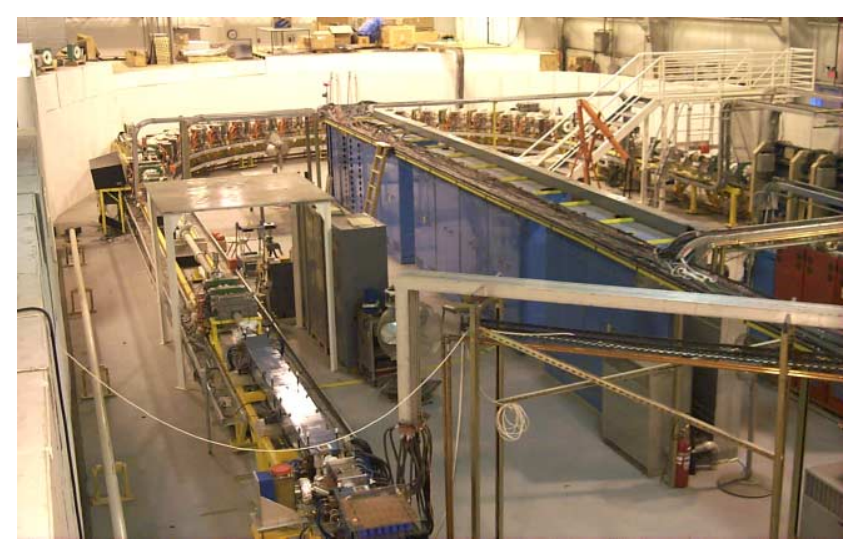

Fig. 1: The west part of the Duke Storage Ring

\section{MACHINE UP-GRADE AND USER APPLICATIONS}

The Duke FEL facility is constructed and operated by a small team, which includes 7 scientists, 5 engineers, 10 technicians and 3 graduate students. Because of the limited resources and other historical reasons, the machine was started only with very necessary basic components. For example, there was only one kicker magnet for injection and the beam position monitors were not available during commissioning. However we successfully stored beam and ramped it from $230 \mathrm{MeV}$ to $1.1 \mathrm{GeV}$ within days. After the commissioning, we installed other two kickers, improved the injector's performance and raised the injection energy to $270 \mathrm{MeV}$. Two years later, the OK-4 FEL was installed and commissioned. The beam position monitors were added to operation 4 years later. Last year, we installed new vacuum crotch chambers at each corner of the ring. The new chambers were designed with synchrotron radiation absorbers and smoother cross-section transitions. This up-grade reduced the ring impedance and allowed us to operate to higher beam current.

This year we have planned to replace all the quadrupole magnet coils and related power supplies. The old coils have overheating problem because of the poor designed cooling system. The current magnet power supplies do not meet the specifications. All the hardware for this upgrade has been acquired. The installation is scheduled in fall.

Next year, we plan to install the OK-5 FEL. It has a 24meter long helical electromagnetic wiggler. This will increase the FEL gain by a factor of 4 and expand the FEL wavelength further down to the VUV and soft X-ray range. The OK-5 can also produce circularly polarized VUV and gamma rays, which are useful for some users. The OK-5 wiggler and its vacuum chamber are under construction at the Budker Institute of Nuclear Physics, Novosibirsk, Russia.

When we try to enhance our machine's performance and capabilities, we also make the efforts to provide useful beam to our users. The user application program started in April 1998. Since then, we managed to have at least half of the time each year for user operation. In 1999, a new user building, adjacent to the main building, was put in use. It has 13,000 SF areas available for users. Currently six experiment stations are set-up. A fully radiation shielded gamma ray vault equipped with gamma ray detectors has been constructed inside the user building. Two optical beam delivery systems have been completed. Both are equipped with a number of beam ports to serve different users. There are two groups of user programs carried out on our facility. One is the gamma ray users for nuclear physics studies. They use about half of the user operation time. Another half of the time is operated for synchrotron and spontaneous radiation users. Their experiments are in the fields of photon electron emission microscope, cornea surgery, material science etc. A third beam line for biological physics and cell biology study is being commissioned at this time.

\section{FUTURE PLAN}

We plan to add a synchrotron booster between the linac injector and the storage ring. The purpose is to enable the full energy injection without ramping. The ramping program, currently used on the ring, works reasonably well but it prevents us from operating at a constant beam current by using frequent injection at the operation energy. We have demonstrated the top-up operation ability at the injection energy of $270 \mathrm{MeV}$. The nearly constant laser beam was delivered by keeping injection manually for 8 hours. With the proposed synchrotron booster, we will be able to do this at any desired operation energy, which will allow us to maintain the gamma flux and the laser beam at a desired level for users.

\section{ACKNOWLEDGEMENTS}

The authors wish to acknowledge every staff member, who worked and works at Duke FEL laboratory. This project takes a whole team working together for many years to reach the current status. Special thanks go to our funding agency: the DOD MFEL program as administrated by the AFOSR, for many years of support.

\section{REFERENCES}

[1] I.Pinayev et al, "Status of the Mark III FEL", these proceedings.

[2] V. N. Litvinenko et al, Proc. of Pac'99, 1999, P.221

[3] Y. Wu et al. "Dynamic Aperture Study for the Duke FEL Storage Ring", $\left(14^{\text {th }}\right.$ Int. FEL Conf., Kobe, Japan, 1992) Nucl. Instr. Meth.

[4] I. V. Pinayev et al, AIP Proc., CP451, 1998, 545 